\title{
Electronic Structure and Luminescence Properties of Er Doped ZnO Nanowires
}

\author{
Juan Wang*, S. K. Hark*, and Quan Li* \\ *Department of Physics, The Chinese University of Hong Kong, Shatin, New Territory, Hong \\ Kong
}

The bottom-up approach utilizing one-dimensional semiconducting nanostructures for future photonic devices becomes one of the most promising methods as it maintains the same concepts as in microeletronics and can potentially overcome the limitations of conventional lithography-based fabrication. Nevertheless, real device application would require effective doping of these nanowires, which process are known to modify their electronic structure and thus the luminescence properties. Er doping into different semiconducting materials are of continuous research interests due to its effective $1.54 \mu \mathrm{m}$ emission for applications in optical communications [1]. Among the semiconductor host, $\mathrm{ZnO}$ is a promising candidate because of its $\sim 3.3 \mathrm{eV}$ wide-band gap and the increasing emission efficiency of Er ions due to oxygen incorporation [2]. In addition, $\mathrm{ZnO}$ itself has already been widely used in the piezoelectric and optoelectronic devices. Although abundant literature results are available regarding the $\mathrm{Er}$ doping into $\mathrm{ZnO}$ thin films, little is known when such doping takes place in nanowires. It is understood from the thin film studies that the $1.54 \mu \mathrm{m}$ luminescence mechanism would occur when the Er atoms are triply ionized and embedded in the $\mathrm{ZnO}$ hosts, allowing an effective energy transfer from the excited host electrons in the conduction band to the Er ions. However, in the case of $\mathrm{ZnO}$ nanowires, it is not clear what kind of dopant sites that Er would take, and how should it affect the $1.54 \mu \mathrm{m}$ emission.

In the present study [3], we demonstrate, by combined microscopy and optical characterizations, that $\mathrm{Er}$ can be effectively doped into $\mathrm{ZnO}$ nanowires via ion implantation. Figure 1 (a) shows a dark filed image of the Er doped $\mathrm{ZnO}$ nanowire, taking by a high angle annular dark field detector. The bright contrasts in the nanowire suggest regions of higher Er concentration. The Er atoms are found to take the substitutional sites in the host lattice. Although no lattice distortion is detected in the host material, electron energy loss spectroscopy (Figure 1 (b)) performed on single $\mathrm{ZnO}$ nanowire reveals defect state(s) in the band gap of the host $\mathrm{ZnO}$ as induced by the Er doping, while the band gap threshold and other single electron excitations of the host remain identical to those of un-doped $\mathrm{ZnO}$ nanowire. Such mid-gap defect band remains even after oxygen annealing of the doped nanowires. The as-implanted $\mathrm{ZnO}$ nanowires give a weak band edge emission and a strong green emission, which is attributed to O-related defects in the nanowires. No emission at $1.54 \mu \mathrm{m}$ is detected. As a comparison, the green emission of the $\mathrm{ZnO}$ host is significantly suppressed in the annealed nanowires, and the room-temperature $1.54 \mu \mathrm{m}$ emission is observed (Figure 1 (c)). All of the experimental results strongly suggest that effective energy transfer from the excited host valence electrons in the conduction band to Er ions contributes to the $1.54 \mathrm{~mm}$ emission. On the other hand, the substitutional $\mathrm{Er}$ ions themselves lead to the generation of lattice point defects in the $\mathrm{ZnO}$ nanowire host, which is detrimental to the $1.54 \mu \mathrm{m}$ emission efficiency.

References 
[1] X. Zhao, S. Komuro, H. Isshiki, Y. Aoyagi, and T. Sugano, Appl. Phys. Lett. 74, (1999) 120. [2] A. Polman, J. Appl. Phys. 82, (1997) 1.

[3] This work is supported by a grant from the the GAESand the RGC of the Hong Kong Joint Research Scheme (project No. G_HK013/05). The authors are indebt to Dr. D. Tang for his help with the STEM and valuable experimental suggestions. QLi Thanks Prof. C.S. Chen and Dr. M.W. Chu for the opportunity to spend time in the National Taiwan University.

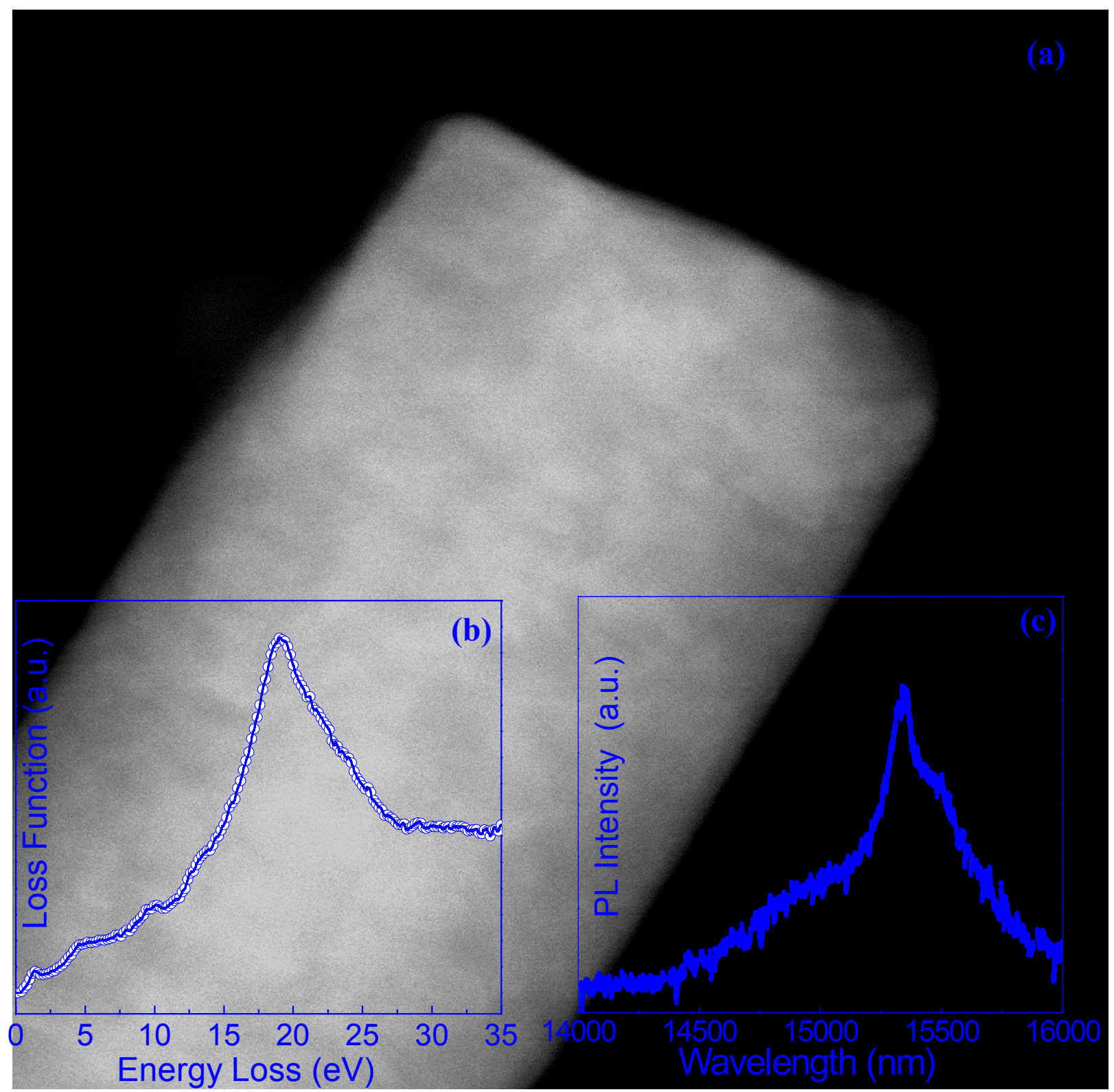

Fig 1. (a) Low magnification STEM image of the Er-doped ZnO nanowire. The light/dark contrast suggests the higher Er concentration region; (b) Loss function of the Er-doped $\mathrm{ZnO}$ nanowire, a $\sim 2 \mathrm{eV}$ mid-gap state is observed in the spectrum; (c) Photoluminescence of the Erdoped $\mathrm{ZnO}$ nanowire, demonstrating the $1.54 \mu \mathrm{m}$ emission. 